\title{
Vohwinkel syndrome: ichthyosiform variant in a family*
}

\author{
Clarissa Prieto Herman Reinehr ${ }^{1}$, Juliano Peruzzo ${ }^{2}$, Tania Cestari ${ }^{2}$
}

DOI: http:/ / dx.doi.org/10.1590/abd1806-4841.20187440

\begin{abstract}
Vohwinkel syndrome belongs to the group of hereditary palmoplantar keratoderma, having an autosomal dominant inheritance. In this report, the authors present a case of a four-year-old boy with diffuse scaling over his entire body and transgredient palmoplantar hyperkeratosis with some fissured areas. Family evaluation revealed that his mother and other family members were affected. Based on his clinical findings and on family history, the diagnosis of the ichthyotic Vohwinkel syndrome subtype, characterized by generalized ichthyosis and palmoplantar hyperkeratosis, was established.
\end{abstract}

Keywords: Genetic diseases, inborn; Ichthyosis; Keratinocytes; Keratoderma, palmoplantar; Skin diseases, genetic

\section{INTRODUCTION}

Vohwinkel syndrome (VS) belongs to the group of hereditary palmoplantar keratoderma and has an autosomal dominant inheritance. ${ }^{1}$ The authors report a case of the ichthyosis subtype in a child (Camisa's syndrome).

\section{CASE REPORT}

A four-year-old boy presented with pruritus and diffuse scaling since birth. The lesions worsen during winter, and the use of moisturizers partially relieves the symptoms. Some relatives had similar experiences (Figure 1). On examination, the patient presented scaling over his entire body and transgredient palmoplantar hyperkeratosis with some fissured areas (Figures 2 and 3). His mother showed similar changes, with honeycomb scales on her body, transgredient palmoplantar hyperkeratosis and digital constricting bands (pseudoainhum) (Figure 4). Based on the patient's clinical findings and his family history, the diagnosis of Vohwinkel syndrome (VS) was established.

\section{DISCUSSION}

Mutilating Palmoplantar Keratoderma, or VS, belongs to the group of the hereditary palmoplantar keratoderma characterized by focal or diffuse palmoplantar thickening. ${ }^{1}$ It starts in childhood and has an autosomal dominant inheritance, occurring predominantly in females and in Caucasian individuals. ${ }^{1}$ VS is characterized by diffuse transgredient palmoplantar hyperkeratosis with a honeycomb appearance, and also affects the extensor surfaces of the limbs. Digi-

Received 24 July 2017

Accepted 10 October 2017

* Work conducted at the Department of Dermatology, Hospital de Clínicas de Porto Alegre, Universidade Federal do Rio Grande do Sul, Porto Alegre (RS), Brasil. Financial support: None.

Conflict of interest: None.

Program of Post-graduation in Medical Sciences, Universidade Federal do Rio Grande do Sul, Porto Alegre (RS), Brazil.

Department of Dermatology, Hospital de Clínicas de Porto Alegre, Universidade Federal do Rio Grande do Sul, Porto Alegre (RS), Brazil.

MAILING AdDREsS:

Clarissa Prieto Herman Reinehr

E-mail: cla.reinehr@gmail.com 


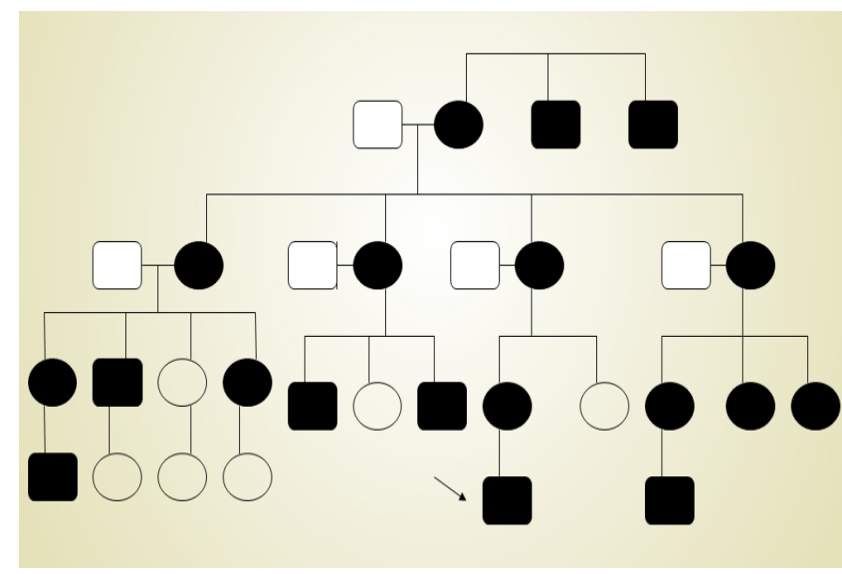

FIGURE 1: Heredogram: family members affected by the syndrome are represented by rectangles (men) or black circles (women). The patient is identified by the black arrow


Figure 2: Patient presenting fine scales throughout body surface $(\mathrm{A}, \mathrm{B})$

tal constricting bands, composed by fibrous connective tissue (pseudoainhum), can lead to autoamputation. Distal starfish-shaped keratotic papules on the dorsum of the feet and hands, wrists, forearms, elbows and knees are also typical findings. ${ }^{1-3}$

Two variants of the syndrome are described: one is associated with ichthyosis (Camisa's syndrome), and the other is accompanied by sensorineural hearing loss. ${ }^{1}$ The ichthyotic variant, which is associated with a mutation in the loricrin gene and results in defective formation of the stratum corneum, is characterized by generalized ichthyosis and palmoplantar hyperkeratosis, as observed in our patient. . $^{2,5}$ Starfish-shaped keratotic papules and deafness are not observed in this variant. ${ }^{1,4}$ The variant with sensorineural hearing loss occurs due to a mutation in the connexin 26 gene (GJB2 gene) and presents with starfish-shaped keratotic papules and palmoplantar hyperkeratosis. Ichthyosis is not seen in this form of the disease. $^{6}$

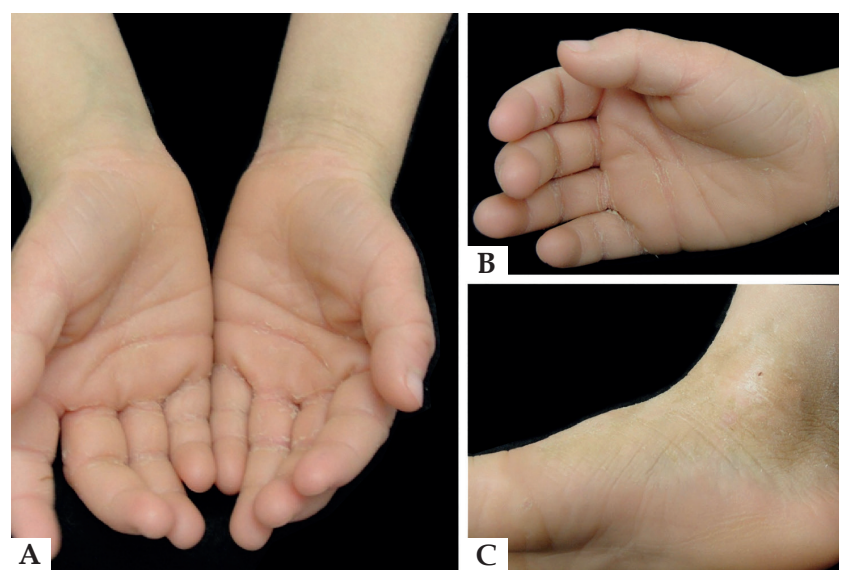

FIGURE 3: Patient with transgredient palmoplantar hyperkeratosis and some palmar fissures (A, B, C)
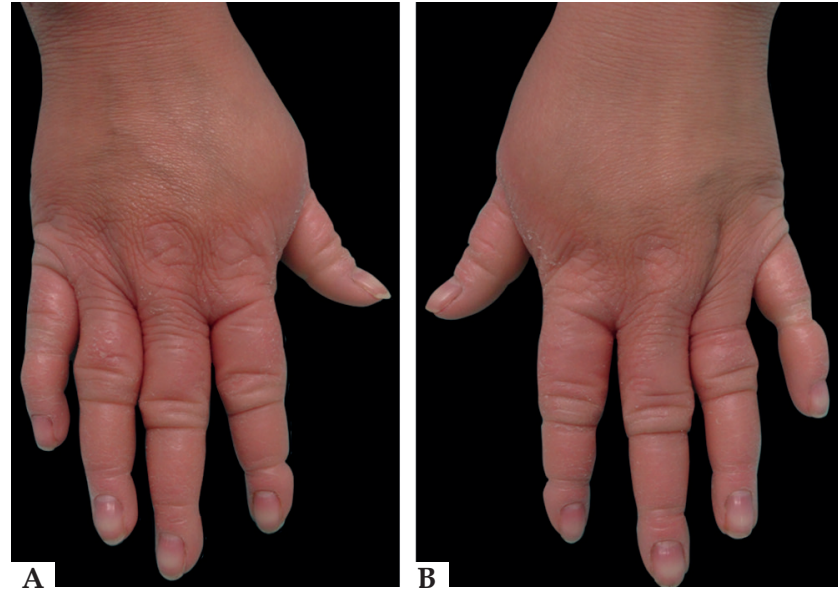

FIGURE 4: Patient's mother presenting digital constrictive bands (pseudoainhum)

The differential diagnosis of VS includes other keratodermas presenting with digital autoamputation, such as Mal de Meleda, Olmsted syndrome, acral keratoderma, pachyonychia congenita, palmoplantar keratoderma of Sybert, and palmoplantar keratoderma of Gamborg-Nielsen, as well as acquired dermatoses that can lead to the appearance of constricting bands, such as leprosy, tertiary syphilis, ainhum, scleroderma, amniotic bands, Raynaud syndrome and syringomyelia. ${ }^{1}$

The aim of treatment is to relieve hyperkeratosis with emollient and keratolytic topical therapy and to prevent autoamputation of digits with therapeutic release of constricting bands. ${ }^{4,7}$ The use of retinoids, such as acitretin, isotretinoin and etretinate, has been demonstrated to result in resolution of pseudoainhum and reversal of keratoderma by decreasing epithelial cell cohesion and inhibiting pathological keratinization. ${ }^{1,8,9}$ 


\section{REFERENCES}

1. Cavalcante LIS, Almeida TLP, Holanda EM, Accioly-Filho JW. Vohwinkel's mutilating keratoderma: report of three familial cases. An Bras Dermatol. 2003:78:311-8.

2. Corte LD, Silva MV, Oliveira CF, Vetoratto G, Steglich RB, Borges J. Vohwinkel syndrome, ichthyosiform variant - by Camisa - Case report. An Bras Dermatol. 2013;88:206-8.

3. Al Aboud K, Al Hawsawi K, Ramesh V. Bilateral Pseudoainhum in Lammelar Ichthyosis. Pediatr Dermatol. 2004;21:181.

4. O'Driscoll J, Muston GC, McGrath JA, Lam HM, Ashworth J, Christiano AM. A recurrent mutation in the loricrin gene underlies the ichthyotic variant of Vohwinkel syndrome. Clin Exp Dermatol. 2002;27:243-6.
5. Ishida-Yamamoto A. Loricrin keratoderma: a novel disease entity characterized by nuclear accumulation of mutant loricrin. J Dermatol Sci. 2003;31:3-8.

6. Dippold S, Butsch F, Schopf R, Keilmann A. Vohwinkel syndrome. Hearing loss and keratoderma on the hands and feet. HNO. 2013;61:617-9.

7. Bassetto F, Tiengo C, Sferrazza R, Belloni-Fortina A, Alaibac M. Vohwinkel syndrome: treatment of pseudo-ainhum. Int J Dermatol. 2010;49:79-82.

8. Nico MMS, Fernandes JD. Low-dose isotretinoin prevents digital amputation in loricrin keratoderma (Vohwinkel syndrome with ichthyosis). J Dtsch Dermatol Ges. 2017;15:665-7.

9. Kura MM, Parsewar S. Reversal of pseudo-ainhum with acitretin in Camisa's syndrome. Indian J Dermatol Venereol Leprol. 2014;80:572-4.

\section{AUTHORS'CONTRIBUTIONS}

Clarissa Prieto Herman Reineh

D ORCID 0000-0003-1811-4519

Approval of the final version of the manuscript, Design and planning of the study, Preparation and writing of the manuscript, Critical review of the literature

Juliano Peruzzo

(iD) ORCID 0000-0001-6963-7921

Approval of the final version of the manuscript, Design and planning of the study, Preparation and writing of the manuscript, Intellectual participation in propaedeutic and/or therapeutic conduct of studied cases, Critical review of the literature, Critical review of the manuscript

\section{Tania Cestari} ORCID 0000-0003-3001-0202

Approval of the final version of the manuscript, Design and planning of the study, Preparation and writing of the manuscript, Effective participation in research orientation, Intellectual participation in propaedeutic and/or therapeutic conduct of studied cases

How to cite this article: Reinehr CPH, Peruzzo J, Cestari T. Vohwinkel syndrome: ichthyosiform variant in a family. An Bras Dermatol. 2018;93(5):723-5. 